Satoru Matsuda $\cdot$ Chisako Iriyama $\cdot$ Shouichi Yokozaki Yasukatu Ichigotani • Naoki Shirafuji • Kenichi Yamaki Tetsuo Hayakawa $\cdot$ Michinari Hamaguchi

\title{
Cloning and sequencing of a novel human gene that encodes a putative target protein of Nesh-SH3
}

Received: March 12, 2001 / Accepted: April 23, 2001

\begin{abstract}
By using a conventional two-hybrid technique with an Src homology 3 (SH3) domain of Nesh as the bait protein, a novel full-length cDNA was isolated and sequenced from a human placenta cDNA library. This cDNA consists of $3023 \mathrm{bp}$ and has a predicted open reading frame that encodes 486 amino acids. It possesses an SH3 binding motif, a nuclear targeting sequence, and no catalytic domain. Overall, it has no similarity to known molecules involved in a signaling cascade. Polymerase Chair reaction-based mapping with both a monochromosomal hybrid panel and radiation hybrid cell panels localized the gene on human chromosome $3 \mathrm{q} 12$ near the marker D3S1271.
\end{abstract}

Key words Two-hybrid $\cdot$ Nesh $\cdot \mathrm{SH} 3 \cdot$ Chromosome $3 \mathrm{q} 12 \cdot$ RH mapping

\section{Introduction}

Src homology 3 (SH3) is a small domain containing about 50 amino acid residues that bind to proline-rich sequences of proteins. Biological processes often require such specific protein-protein interactions in the formation of signaling complexes (Buday 1999). In this article we describe the

S. Matsuda $(\bowtie) \cdot$ C. Iriyama $\cdot$ Y. Ichigotani $\cdot$ M. Hamaguchi Department of Molecular Pathogenesis, Nagoya University School of Medicine, 65 Tsurumai-cho, Showa-ku, Nagoya 466-8550, Japan Tel. +81-52-744-2463; Fax +81-52-735-8167

e-mail: smatsuda@med.nagoya-u.ac.jp

S. Yokozaki $\cdot$ K. Yamaki $\cdot$ T. Hayakawa

Second Department of Internal Medicine, Nagoya University School of Medicine, Nagoya, Japan

N. Shirafuji

Internal Medicine, Teikyo University School of Medicine, Tokyo, Japan

The nucleotide sequence data reported in this article have been deposited with the DDBJ, EMBL, and GenBank data libraries under accession number AB056106. cloning and characterization of a novel human gene that encodes a predicted protein with a consensus sequence of SH3 binding. We named the molecule Tarsh because it seemed to be a putative target of Nesh- $\underline{\mathrm{H}} 3$. Nesh was recently identified as a novel protein containing an SH3 domain and proline-rich sequences similar to e3B1 (Miyazaki et al. 2000), whose biochemical and physiological role has not yet been clarified. The identification and characterization of a binding protein to Nesh is necessary to elucidate further precise mechanisms of its cellular function. Accordingly, we tried to identify potential Nesh partners to gain an understanding of Nesh function. The use of the yeast two-hybrid system enabled us to isolate the Tarsh gene. The interaction has not yet been characterized in detail by other commonly used protein-binding assays. However, because Tarsh has never been described before, we were prompted to further characterize this gene.

\section{Methods, results, and discussion}

We used the SH3 domain (corresponding to amino acids 294-366) of human Nesh for bait plasmids in the yeast twohybrid screens, and identified a clone from a cDNA library constructed from human placenta poly $(\mathrm{A})^{+}$RNA. The clone cDNA was reconfirmed to be interaction-positive by using an independent yeast clone containing the bait. DNA sequencing of the insert region was then performed by using a Sequenase Kit (Amersham, Cleveland, OH, USA) and an automatic sequencer for dideoxy sequencing (Amersham) according to the supplier's instruction. We used the insert nucleotide sequence as a probe for further screening to yield cDNAs encoding full-length and for searching the gene database to confirm the sequence and the gene structure (Matsuda et al. 1996; Matsuda et al. 2000a; Matsuda et al. 2000b; Miyazaki et al. 2000).

The determined nucleotide sequence and predicted amino acid sequence are shown in Fig. 1. The cDNA of $3023 \mathrm{bp}$ contains an uninterrupted open reading frame of $1458 \mathrm{bp}$ and a poly(A) tail. The Tarsh protein was thus 
Fig. 1. Representation of the Tarsh cDNA and sequences. Nucleotide and predicted amino acid sequences of the Tarsh gene. The complete sequence of the cDNA was determined and confirmed by the primer walking strategy with dideoxy sequencing. The nucleotide sequence data have been submitted to the GenBank and EMBL data libraries under accession number AB056106. Numbering of the nucleotide and amino acid sequences is shown on the right and left, respectively. Polyadenylation signals (AATAAA) exist in the 3 '-noncoding DNA sequence (nucleotides 2772-2777 and 29592964). The original sequence of the two-hybrid positive clone started from nucleotide 141 and ended at nucleotide 3023. The Src homology 3 (SH3) binding consensus sequence (amino acids 117-122) is indicated by a shaded box, and the predicted bipartite nuclear targeting sequence (amino acids 116-130) is boldfaced
CCATTTAATGTATAGTTTATT TCTAGGTTTTCCTTCTGTGTTTTTAGACACTGAAATGCCTAAATATTGATTTGTTATTATTTTGTTC TTTATT TTTCCAGCTCCCGGTAAAACACAAT TTAT T TCTCTGAAACCTAAAATCCCTCTCAGCCCAGAAGTGACACACACCAAACCTGCT CCCAAGCAGACACCACGTGCTCCTCCTAAGCCAAAAACATCACCACGCCCAAGAATCCCACAAACACAACCAGTTCCTAAGGTGCCCCAG CGTGTTACTGCAAAACCAAAAACGTCACCAAGTCCAGAAGT GTCATACACCACACCTGCTCCTCTAGAGACACGAGGCATCCCTTTTATA CCCATGATT TCCCCAAGTCCTAGTCAAGAGGACTACAGACCACTCTGGAAGAAACAGACCAATCCACCCAAGAACCTT TCACAACTAAG

$\begin{array}{llllllllllllllllllllllllllllll}M & I & S & P & S & P & S & Q & E & E & L & Q & T & T & L & E & E & T & D & Q & S & T & Q & E & P & F & T & T & R\end{array}$ ATTCCACGAACAACTGAACTAGCAAAGACAACTCAGgCGCCACACAGATTTTATACTACTGTGAGGCCAGAaCATCTGACAagCCACAC 540

$\begin{array}{llllllllllllllllllllllllllllllll}30 & I & P & R & T & T & E & \text { L } & A & R & T & T & O & A & P & H & R & F & Y & T & T & V & R & P & R & T & S & D & R & P & B\end{array}$ ATCAGACCTGTTCTGAATAGGACAACTACAAGACCTACTAGGCCCAAACCCAGTGGGATGCCCAGTGGGATGGAGTGGGACAGGGGTC

$\begin{array}{lllllllllllllllllllllllllllllllll}60 & \text { I } & R & P & V & \text { L } & N & R & T & T & T & R & P & T & R & P & K & P & S & G & M & P & S & G & N & G & V & G & T & G & V\end{array}$ AAGCAAGCACCCAGGCCATCAGGTGCTGATAGAAATGTATCAGTGGACTCTACCCACCCCACTAAAAAGCCAGGGACTCGCCGCCCACCC

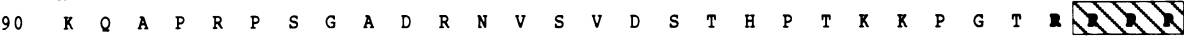
TTGCCACCCAGACCTACACACCCACGAAGAAAACCTTTACCACCAAATAATGTCACTGGAAAGCCAGGAagTGCAGgAaTCATTTCATCA

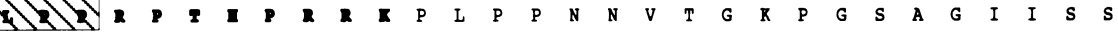
GGCCCAATAACTACACCACCCCTGAGGTCAACACCCAGGCCTACTGGAACTCCCTTGGAGAGAATAGAGACAGATATAAagCAACCAACA

$\begin{array}{lllllllllllllllllllllllllllllllllll}150 & G & P & I & T & T & P & P & I & R & S & T & P & R & P & T & G & T & P & L & E & R & I & E & T & D & I & R & Q & P & T\end{array}$ GTTCCTGCCTCTGGAGAAGAACTGGAAATATAACTGACTTTAGCTCAAGCCCAACAAGAGAAACTGATCCTCTTGGGAaGCCAAGATTC 990

$\begin{array}{llllllllllllllllllllllllllllllll}180 & V & P & A & S & G & E & E & L & E & N & I & T & D & F & S & S & S & P & T & R & E & T & D & P & L & G & R & P & R & P\end{array}$ AAAGGACCTCATGTGCGATACATCCAAAAGCCTGACAACAGTCCCTGCTCCATTACTGACTCTGTCAAACGGTTCCCCAAAGAGGAGCC 1080

$\begin{array}{lllllllllllllllllllllllllllllllll}210 & K & G & P & H & V & R & Y & I & Q & K & P & D & N & S & P & C & S & I & T & D & S & V & K & R & F & P & R & E & E & A\end{array}$ ACAGAGGGGAATGCCACCAGCCCACCACAGAACCCACCCACCAACCTCACTGTGGTCACCGTGGAAGGGTGCCCTCATTTGTCATCTTG 1170

$\begin{array}{lllllllllllllllllllllllllllllllllll}240 & T & \text { T } & G & N & A & T & S & P & P & Q & N & P & P & T & N & \text { L } & T & V & V & T & V & E & G & C & P & S & F & V & I & L\end{array}$ GACTGGGAAAGCCACTAAATGACACTGTCACTGAATATGAAGTTATATCCAGAGAAAATGGGTCATTCAGTGGGAAGAACAAGTCCATT 1260

$\begin{array}{lllllllllllllllllllllllllllllllll}270 & D & W & E & K & P & \text { L } & N & \text { D } & T & \text { V } & \text { T } & \text { E } & \text { Y } & \text { E } & \text { V } & \text { I } & S & R & E & N & G & S & F & S & G & K & N & K & S & I\end{array}$ CAAATGACAAATCAGACATTTTCCACAGTAGAAAATCTGAAACCAAACACGAGT TATGAATTCCAGGTGAAACCAAAAACCCACTTGGT 1350

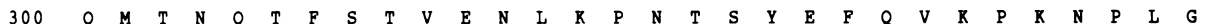
GAAGGCCCGGTCAGCAACACAGTGGCATTCAGTACTGAATCAGCGGACCCAAGAGTGAGTGAGCCAGTTTCTGCAGGAagaGatGCCATC 1440

$\begin{array}{llllllllllllllllllllllllllllllll}330 & E & G & P & V & S & N & T & V & A & F & S & T & E & S & A & D & P & R & V & S & E & P & V & S & A & G & R & D & A & I\end{array}$ TGGACTGAAAGACCCTTTAATTCAGACTCTTACTCAGAGTGTAAGGCAAACAATATGTCAAAAGGACATGGTATAAAAAATTGTAGGA 1530

$\begin{array}{llllllllllllllllllllllllllllllllll}360 & \text { W } & T & \text { E } & R & P & F & N & S & D & S & Y & S & E & C & R & G & K & Q & Y & V & \mathbb{R} & R & T & W & Y & R & R & F & V & G\end{array}$ GTGCAGCTGTGCAACTCTCTCAGATACAAGATTTACTTGAGCGACTCCCTCACAGGAAATTTTATAACATAGGTGATCAGAGGGCCAT 1620

$\begin{array}{llllllllllllllllllllllllllllllllllll}390 & V & Q & L & C & N & S & L & R & Y & K & I & Y & L & S & D & S & L & T & G & R & F & Y & N & I & G & D & Q & R & G & B\end{array}$ GGAGAAGATCACTGCCAGTTTGTGGATTCATTTTTAGATGGACGCACTGGGCAGCAACTCACTTCTGACCAGTTACCAATCAAAGAAGT 1710

$\begin{array}{llllllllllllllllllllllllllllllllllll}420 & G & E & D & H & C & Q & F & V & D & S & F & L & D & G & R & T & G & Q & Q & L & T & S & D & Q & L & P & I & R & E & G\end{array}$ TATTTCAGAGCAGTTCGCCAGGAACCTGTCCAATTTGGAGAAATAGGTGGTCACACCCAAATCAATTATGTTCAGTGGTATGAATGTGG 1800

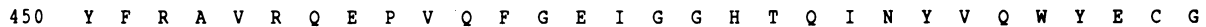
ACTACAATCCTGGAAATGGTAGATGCTGCACAAAgTTACCTTCTGTTTCATCATTGCAAACAAAAATCATTGAAATACTATgCCGCA 1890

480 T T I P G K W *

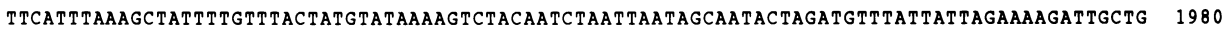
AGAGTATTTATCAGGT T T TACAAAGTCATT T TAAGAAAGCAAGATACTGATGTTAACAGAATAACATTT TTGGGGAAGCTGGCTCCCTAT 2070 TCATGGTATTTAAGAGATCATT T GTATATTATTTATCACACTGTTGTAATGATGTTTTGAGATACTTTTATAACAAATTAACATCAAA 2160 AAGGTATATACT TTTTAAAAAAAATTTACTTTTATTGATGTGTACTCTTCCTATTGATGAGTTAATTCCATAAATCTCTACTTAGTTTAa 2250 CTTATTGGATCAAATTATCTTCAGCATGTATATCTGGGGAAAAAGGTCCGAATTTTCACATTTATATTTAAACTTCAATTTTTATATT 2340 TAAACT TCAATT TTTAGCAACAGCTGAATAGCTTTGCGGAGGAGTTTAATAGTTACACATTCATGCTAATATACATTTCCTTTAAACAT 2430 CCACAAATT T TAAAAAGATTGAATCAGTAAATTTCATTTCAGCTAAAAATGGAGTCTAATATATTGTTTCAAAAGATACATTTTTACCC 2520 ACCATAAATGTTACAATATCTGAATATGCTTTGTCAAACTATCCCTTTATGCAATCGTCTTCATATTGTTTTTATGATTCTAATCAAGCT 2610 GTATGTAGAGACTGAATGTGAAGTCAAGTCTGAGCACAAAAAGATAATGCACGATGAGATTGCCTACCATTTTATAGGATATTTACTATG 2700 TATTTATACGTTAAGACCTCTATGAATGAATGTATCAGAGAATGTCTTTGTAACTGTTTAATTCAATCTGTAATAAAAATCTAACTAACT 2790 AACTCATTTATTTCTATTAAAAAGGTATTGTCCTTTAGGCGGGGAATGGGAATCCTTGCTGCACTGTTGCAGTCATTCTGAAAGGACCTT 2880 TCCCTGTACT TACCT T TCAACATGCTTCAATCTTATCAACGCTACATTT TGTATTTTTCAAACAAGTATAAATTCTGCAATAAAGAGATG 2970 TAGT T T T T T TAAAAAAAAAAAAAAAAAAAAAAAAAAAAAAAAAAAAAAAAAA

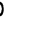

(1)

\section{.} . . ,

\section{,}

\section{.}




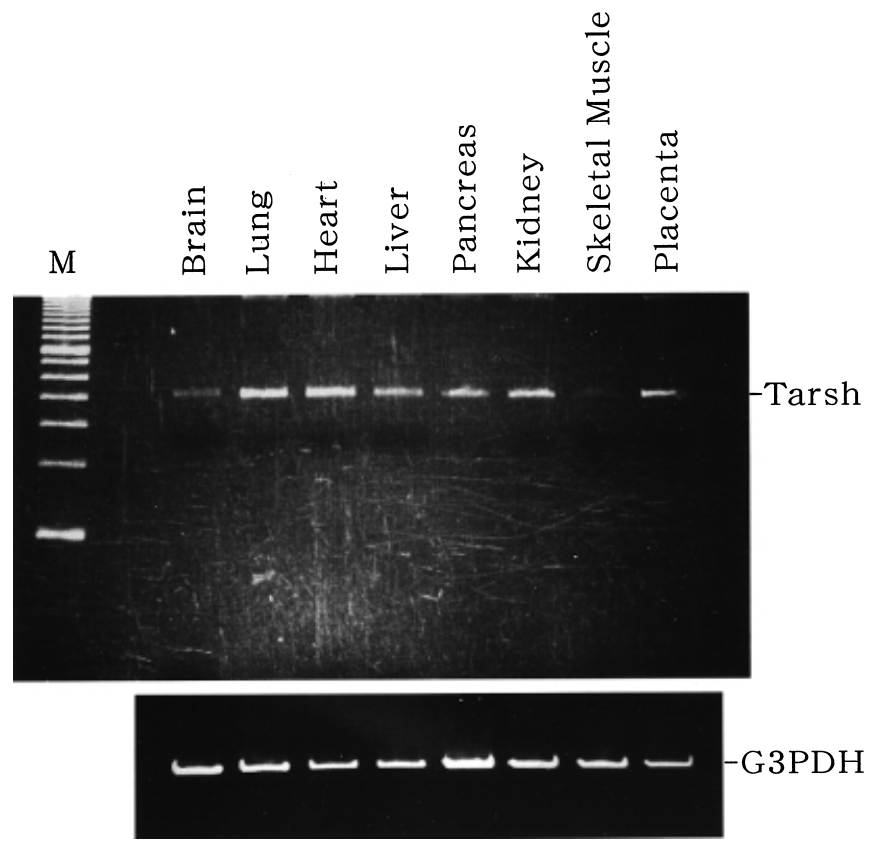

Fig. 2. Expression of the Tarsh gene in multiple normal human tissues. Reverse transcription and amplification by polymerase chain reaction with the specific primers for the Tarsh gene were performed to analyze Tarsh expression (top panel). The eight tissues examined are indicated above each lane. The template cDNA for brain (lane 1), lung (lane 2), heart (lane 3), liver (lane 4), pancreas (lane 5), kidney (lane 6), skeletal muscle (lane 7), and placenta (lane 8) of the human normal tissues were purchased from Clontech (Palo Alto, CA, USA). Lane $M$, marker. The bottom panel shows the expression of glycerol-3-phosphate dehydrogenase $(G 3 P D H)$ that was analyzed as a control

by using cycle-limited RT-PCR ( 28 cycle amplified), expression of Tarsh mRNA was detected at significant levels in several adult tissues (brain, heart, lung, liver, pancreas, kidney, and placenta) but not in skeletal muscle. This result suggests that Tarsh might be ubiquitously expressed except for skeletal muscle, although the level of expression varies. In this experiment genomic DNA might not be amplified, because primers designed from the noncoding region of Tarsh could not be amplified (data not shown). Considering its expression profile, Tarsh might have a fundamental function in a broad spectrum of adult tissue cells.

To determine the chromosomal localization of the Tarsh gene, we performed PCR-based monochromosomal somatic cell hybrid mapping (Quantum, Montreal, QC, Canada) with a set of $3^{\prime}$ untranslated region-primers (5'TGCTGCACAAAGTTAC- $3^{\prime}$ and $5^{\prime}$-CATCTAGTATTG CTAT-3'). This study indicated that the Tarsh gene is located on human chromosome 3 (Fig. 3A). To further refine the subchromosomal location of the gene, we used radiation hybrid (RH) panels (Stanford G3 and Genebridge 4). Both of the linkage analyses of the PCR results (the data vector for Tarsh of the Stanford G3 and the Genebridge 4RH panels: 10000000000000000000000000101000020 110001001000001000010000000010000100010001010 000 and 0000010110011000111101000001100100010100 110010001011101000110110010010101010001000001 01010100 , respectively) showed consistently that the Tarsh
A

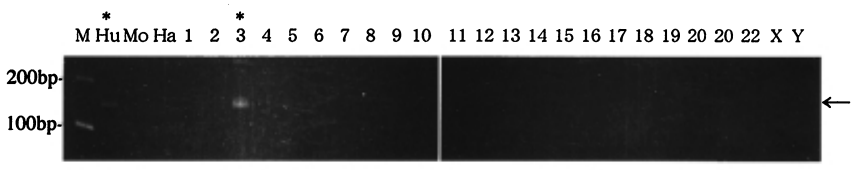

B

\section{Chromosome 3}

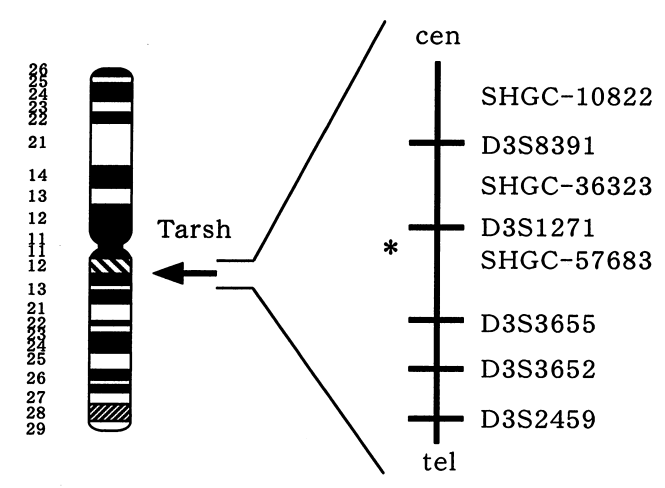

Fig. 3. Chromosomal assignment of the Tarsh gene. A Representative polymerase chain reaction (PCR)-based monochromosomal somatic cell hybrid mapping of Tarsh. Primers from the $3^{\prime}$ untranslated region of the Tarsh cDNA were used. Lanes 1-22 and lanes $X$ and $Y$ represent human chromosomes. Lanes $H u, M o$, and $H a$ represent cell hybrids containing genomic DNA from human, mouse, and hamster, respectively. A single product of the expected size was generated from chromosome 3 and human genomic DNA (indicated by asterisk). Lane $M$, size marker. The arrow indicates the expected size of PCR product. B Chromosomal localization of the Tarsh gene in a schematic ideogram of human chromosome 3 . The approximate corresponding cytogenetic location of the gene on chromosome $3 \mathrm{q} 12$ by the results of radiation hybrid panel mapping is shown. The asterisk shows the putative localization of the Tarsh gene. Cen, Centromere; tel, telomere

gene was linked to several markers adjacent to marker D3S1271 in chromosome 3q12 with logarithm of differences score values higher than 16.

The most likely order of the refined loci is shown in Fig. 3B. Other genes that have been mapped to chromosome 3q12 near the Tarsh gene include the sporadic parathyroid adenoma gene (Thompson et al. 1995) and the tumor initiation and progression gene of multiple endocrine neoplasia type 2 (Mulligan et al. 1993). Although there is no evidence of Tarsh involvement in the genesis of these diseases, our precise chromosomal positioning data contributes a positional candidate approach for the disease genes linked to this locus. Future studies will address the biological role of Tarsh with reference to Nesh binding to understand the function of Tarsh in pathogenesis.

Acknowledgments This work was supported in part by a Grant-in-Aid for Scientific Research on Priority Areas and for COE Research from the Ministry of Education, Science and Culture of Japan, a Grant under the Monbukagakusho International Scientific Research Program. We thank Yu-ki Iwata for her excellent technical assistance. 


\section{References}

Buday L (1999) Membrane-targeting of signalling molecules by SH2/ SH3 domain-containing adaptor proteins. Biochim Biophys Acta 1422:187-204

Kozak M (1989) The scanning model for translation: an update. J Cell Biol 108:229-241

Matsuda S, Kawamura-Tsuzuku J, Ohsugi M, Yoshida M, Emi M, Nakamura Y, Onda M, Yoshida Y, Nishiyama A, Yamamoto T (1996) Tob, a novel protein that interacts with p185erbB2, is associated with anti-proliferative activity. Oncogene 12:705-713

Matsuda S, Katsumata R, Okuda T, Yamamoto T, Miyazaki K, Senga T, Machida K, Thant AA, Nakatsugawa S, Hamaguchi M (2000a) Molecular cloning and characterization of human MAWD, a novel protein containing WD-40 repeats frequently overexpressed in breast cancer. Cancer Res 60:13-27

Matsuda S, Ichigotani Y, Okuda T, Irimura T, Nakatsugawa S, Hamaguchi M (2000b) Molecular cloning and characterization of a novel human gene (HERNA) which encodes a putative RNAhelicase. Biochim Biophys Acta 1490:163-169

Matsuda S, Miyazaki K, Ichigotani Y, Kurata H, Takenouchi Y, Yamamoto T, Nimura Y, Irimura T, Nakatsugawa S, Hamaguchi M (2000c) Molecular cloning and characterization of a novel human gene (NESCA) which encodes a putative adapter protein containing SH3. Biochim Biophys Acta 1491:321-326

Miyazaki K, Matsuda S, Ichigotani Y, Takenouchi Y, Hayashi K, Fukuda Y, Nimura Y, Hamaguchi M (2000) Isolation and characterization of a novel human gene (NESH) which encodes a putative signaling molecule similar to e3B1 protein. Biochim Biophys Acta 1493:237-241

Mulligan LM, Gardner E, Smith BA, Mathew CG, Ponder BA (1993) Genetic events in tumour initiation and progression in multiple endocrine neoplasia type 2. Genes Chromosomes Cancer 6:166-177

Robbins J, Dilworth SM, Laskey RA, Dingwall C (1991) Two interdependent basic domains in nucleoplasmin nuclear targeting sequence: identification of a class of bipartite nuclear targeting sequence. Cell 64:615-623

Seki N, Hattori A, Hayashi A, Kozuma S, Ohira M, Hori T, Saito T (1999) Structure, expression profile and chromosomal location of an isolog of DNA-PKcs interacting protein (KIP) gene. Biochim Biophys Acta 1444:143-147

Thompson DB, Samowitz WF, Odelberg S, Davis RK, Szabo J, Heath H (1995) Genetic abnormalities in sporadic parathyroid adenomas: loss of heterozygosity for chromosome $3 \mathrm{q}$ markers flanking the calcium receptor locus. J Clin Endocrinol Metab 80:3377-3380 